\title{
IMPROVED BICHARACTERISTIC SCHEMES FOR TWO-DIMENSIONAL ELASTODYNAMIC EQUATIONS
}

\author{
BY
}

\author{
X. LIN AND J. BALLMANN
}

\section{Lehr-und Forschungsgebiet für Mechanik der RWTH Aachen, Germany}

\begin{abstract}
Several authors have used explicit numerical schemes of bicharacteristics to solve the system of hyperbolic partial differential equations describing the two-dimensional propagation of stress waves in elastic solids. However, their numerical approaches differ slightly, which results in different limits of the CFL number for stable solutions and in different defects of numerical accuracy near singular points like, e.g., a numerically caused cutting trace emanating from a crack tip. After reviewing the different approaches, some techniques are presented to set up stable explicit schemes with CFL number up to the limiting values 1 for the fastest mode. Finally, the schemes are applied to the crack problem of a shock loaded body, where the main reasons for the appearance of a cutting trace become apparent.
\end{abstract}

1. Introduction. Stress waves in elastic solids, which may be caused by an impact or other impulsive loading, are governed by a system of hyperbolic partial differential equations. Hyperbolic PDEs exhibit undetermined derivatives in certain space-time directions, which define the normals of so-called characteristic manifolds. These manifolds represent singular surfaces along which the disturbances propagate continuously while certain derivatives in the normal direction may be discontinuous. Thus every sophisticated numerical method for the solution of hyperbolic PDEs has to employ the directions of wave propagation in some way. Methods of bicharacteristics use these directions explicitly and have therefore a direct physical interpretation. Consequently it might be expected that those methods represent a good tool for the understanding of transient stress fields and dynamic fracture processes in solids generated by impulsive loading.

Methods of characteristics were introduced first in gas dynamics for inviscid compressible flows where scalar constitutive equations govern the material behaviour and pressure disturbances propagate along characteristics. Clifton [1] transmitted the method of bicharacteristics, which was developed originally by other authors for multi-dimensional unsteady flows, to the two-dimensional propagation of stress waves in isotropic linear elastic bodies with straight boundaries. In contrast to an

Received December 9, 1992.

1991 Mathematics Subject Classification. Primary 73C05, 73D99, 73V15; Secondary 35L05.

This paper is dedicated to Professor Alan Jeffrey on the occasion of his 65 th birthday.

(C)1995 Brown University 
inviscid, compressible fluid, due to the tensor constitutive law in an elastic solid two kinds of waves occur, a longitudinal one and a transverse one.

Since Clifton's paper, various forms of explicit bicharacteristic schemes have been introduced and used by several authors to deal with linear and nonlinear elastic problems [2-5] and elastic-viscoplastic problems [6-12]. Many important results were obtained, e.g., for focusing of waves in plates with curved boundaries, for the dynamic stress intensity factors at crack tips, for stress wave interactions at material interfaces, and steepening and focusing caused by nonlinearity.

However, there are still two major problems arising in the application. One is caused by the discrepancy of the numerical and physical wave speeds, described by the CFL number (Courant-Friedrichs-Lewy), which produces numerical dispersion and-by interpolation procedures in the initial values plane-numerical viscosity, too. All above-mentioned schemes are explicit and use only a single numerical mesh in space with mainly quadratic cells. Under these conditions it is at most possible to achieve the parity of numerical and physical wave speeds for the fastest wave, i.e., the longitudinal one. Accordingly, the CFL number is based on the longitudinal wave speed $c_{1}$. In order to obtain an appropriate numerical approximation with only moderate numerical dispersion and dissipation, one should have $\mathrm{CFL}=1$ at least for this mode. However, in [1] and [6-12], CFL was set to 0.5 (in [7] even 0.25) for the sake of the $\mathbf{J}$. von Neumann stability condition. The other problem is connected with singular points like crack tips or points at the boundary where the prescribed conditions include a discontinuity. It may happen that the singularity seems to be continued by the numerical scheme as a cutting trace through the solution domain, which represents a nonphysical discontinuity of the stress solution. It has been shown in [13] that the dimensional splitting technique [14] will cause such a cutting trace. Nevertheless, cutting traces also appeared in the results of [5] and [12]. This fact means that the cutting trace problem exists for the bicharacteristic method, too.

In this paper, we consider the two above-mentioned problems. Firstly, a general expression of bicharacteristic solutions for the elastodynamic equations is presented. Then, based on this expression, some finite difference schemes are presented, where the main interest is focused on the von Neumann stability condition in the case $\mathrm{CFL}=1$. Finally, the capabilities of different schemes in treating singular point problems are discussed on the basis of numerical examples.

2. Basic equations and bicharacteristic solution. The elastodynamic equations for an isotropic linear elastic solid under plane strain can be written in the following form:

$$
\begin{gathered}
\frac{\partial \mathbf{w}}{\partial t}=\mathbf{A} \frac{\partial \mathbf{w}}{\partial x}+\mathbf{B} \frac{\partial \mathbf{w}}{\partial y}, \\
\mathbf{w}=\left(\begin{array}{c}
u \\
v \\
p \\
q
\end{array}\right), \quad \mathbf{A}=\left(\begin{array}{ccccc}
0 & 0 & 1 & 0 & 0 \\
0 & 0 & 0 & 0 & 1 \\
1 & 0 & 0 & 0 & 0 \\
a & 0 & 0 & 0 & 0 \\
0 & b^{2} & 0 & 0 & 0
\end{array}\right), \quad \mathbf{B}=\left(\begin{array}{ccccc}
0 & 0 & 0 & 0 & 1 \\
0 & 0 & 0 & 1 & 0 \\
0 & a & 0 & 0 & 0 \\
0 & 1 & 0 & 0 & 0 \\
b^{2} & 0 & 0 & 0 & 0
\end{array}\right),
\end{gathered}
$$

where $u$ and $v$ denote the dimensionless particle velocities in the $x$-and $y$-direc- 
tions, respectively (their dimensional quantities are $u c_{1}$ and $\left.v c_{1}\right) ; p=\sigma_{x} /\left(\rho c_{1}^{2}\right)$, $q=\sigma_{y} /\left(\rho c_{1}^{2}\right)$, and $\tau=\sigma_{x y} /\left(\rho c_{1}^{2}\right)$ are dimensionless stress components; $\rho$ is the density, $c_{1}$ and $c_{2}$ are the longitudinal and transverse wave speeds, $b=c_{2} / c_{1}$, $a=1-2 b^{2} ; x$ and $y$ are Cartesian coordinates, and $t$ represents the time multiplied by $c_{1}$. In some cases it will be convenient to write Eq. (1) in the conservative form

$$
\frac{\partial \mathbf{w}}{\partial t}=\frac{\partial \mathbf{f}}{\partial x}+\frac{\partial \mathbf{g}}{\partial y}
$$

with $\mathbf{f}=\left(p, \tau, u, a u, b^{2} v\right)^{\mathrm{T}}$ and $\mathbf{g}=\left(\tau, q, a v, v, b^{2} u\right)^{\mathrm{T}}$.

The conclusive bicharacteristic relations for Eq. (1) can be obtained by the methods presented in [1] or [2]. However, for a problem with only one straight boundary and a corresponding finite difference scheme with a rectangular mesh, only bicharacteristic lines parallel to the $(x, t)$-plane or the $(y, t)$-plane will be used. In this case, a straightforward approach can be used to obtain the bicharacteristic relations.

Suppose there is a characteristic solution for $\mathbf{w}$ in an $(x, t)$-plane $(y=$ constant $)$; then

$$
\frac{d \mathbf{w}}{d t}=\frac{\partial \mathbf{w}}{\partial t}+c \frac{\partial \mathbf{w}}{\partial x} \quad\left(c=\frac{d x}{d t}\right) .
$$

Combining Eqs. (1) and (3), we get

$$
(\mathbf{A}+c \mathbf{I}) \frac{\partial \mathbf{w}}{\partial x}=\frac{d \mathbf{w}}{d t}-\mathbf{B} \frac{\partial \mathbf{w}}{\partial y},
$$

where $\mathbf{I}$ is a unit matrix. For the characteristic solution, $c$ satisfies

$$
\|\mathbf{A}+c \mathbf{I}\|=0
$$

which leads to

$$
c=\frac{d x}{d t}= \pm 1, \pm b
$$

For the four characteristic values $c= \pm 1, \pm b$, the solutions of equation

$$
\mathbf{1}^{\mathrm{T}}(\mathbf{A}+c \mathbf{I})=\mathbf{0}
$$

are four left-eigenvectors $\mathbf{1}_{ \pm 1}^{\mathrm{T}}=(1,0, \mp 1,0,0), \mathbf{1}_{ \pm b}^{\mathrm{T}}=(0,1,0,0, \mp 1 / b)$. Substituting these left-eigenvectors into the equation,

$$
\mathbf{1}^{\mathrm{T}}\left(\frac{d \mathbf{w}}{d t}-\mathbf{B} \frac{\partial \mathbf{w}}{\partial y}\right)=0,
$$

four compatibility relations along the bicharacteristic lines in the $(x, t)$-plane are obtained:

$$
\begin{aligned}
& \left.\frac{d u}{d t} \mp \frac{d p}{d t}-\frac{\partial \tau}{\partial y} \pm a \frac{\partial v}{\partial y}=0 \quad \text { (along } \frac{d x}{d t}= \pm 1\right), \\
& \frac{d v}{d t} \mp \frac{1}{b} \frac{d \tau}{d t}-\frac{\partial q}{\partial y} \pm b \frac{\partial u}{\partial y}=0 \quad \text { (along } \frac{d x}{d t}= \pm b \text { ). }
\end{aligned}
$$

Similarly, the compatibility relations in a $(y, t)$-plane $(x=$ constant $)$ can be derived as 


$$
\begin{aligned}
& \left.\frac{d v}{d t} \mp \frac{d q}{d t}-\frac{\partial \tau}{\partial x} \pm a \frac{\partial u}{\partial x}=0 \quad \text { (along } \frac{d y}{d t}= \pm 1\right), \\
& \left.\frac{d u}{d t} \mp \frac{1}{b} \frac{d \tau}{d t}-\frac{\partial p}{\partial x} \pm b \frac{\partial v}{\partial x}=0 \quad \text { (along } \frac{d y}{d t}= \pm b\right) \text {. }
\end{aligned}
$$

The finite difference scheme can be obtained by integration of Eqs. (9) and (10) along the bicharacteristic lines. Suppose $\left(x_{0}, y_{0}\right)$ to be inside the considered twodimensional region, and $\mathbf{w}(x, y, t)$ to be given by the initial values in the plane $t=t_{0}$ (see Fig. 1). We want to determine the value of $\mathbf{w}$ at the point $\left(x_{0}, y_{0}, t_{0}+\Delta t\right)$. Starting from this point, we draw eight backward lines according to the bicharacteristic directions given in Eqs. (9) and (10). The points of penetration of these lines with the initial value plane are denoted as follows:
$\langle 1\rangle:\left(x_{0}-\Delta t, y_{0}, t_{0}\right)$
$\langle 1\rangle^{\prime}:\left(x_{0}-b \Delta t, y_{0}, t_{0}\right)$
$\langle 2\rangle:\left(x_{0}+\Delta t, y_{0}, t_{0}\right)$
$\langle 2\rangle^{\prime}:\left(x_{0}+b \Delta t, y_{0}, t_{0}\right)$,
$\langle 3\rangle:\left(x_{0}, y_{0}-\Delta t, t_{0}\right)$,
$\langle 3\rangle^{\prime}:\left(x_{0}, y_{0}-b \Delta t, t_{0}\right)$,
$\langle 4\rangle:\left(x_{0}, y_{0}+\Delta t, t_{0}\right)$,
$\langle 4\rangle^{\prime}:\left(x_{0}, y_{0}+b \Delta t, t_{0}\right)$.

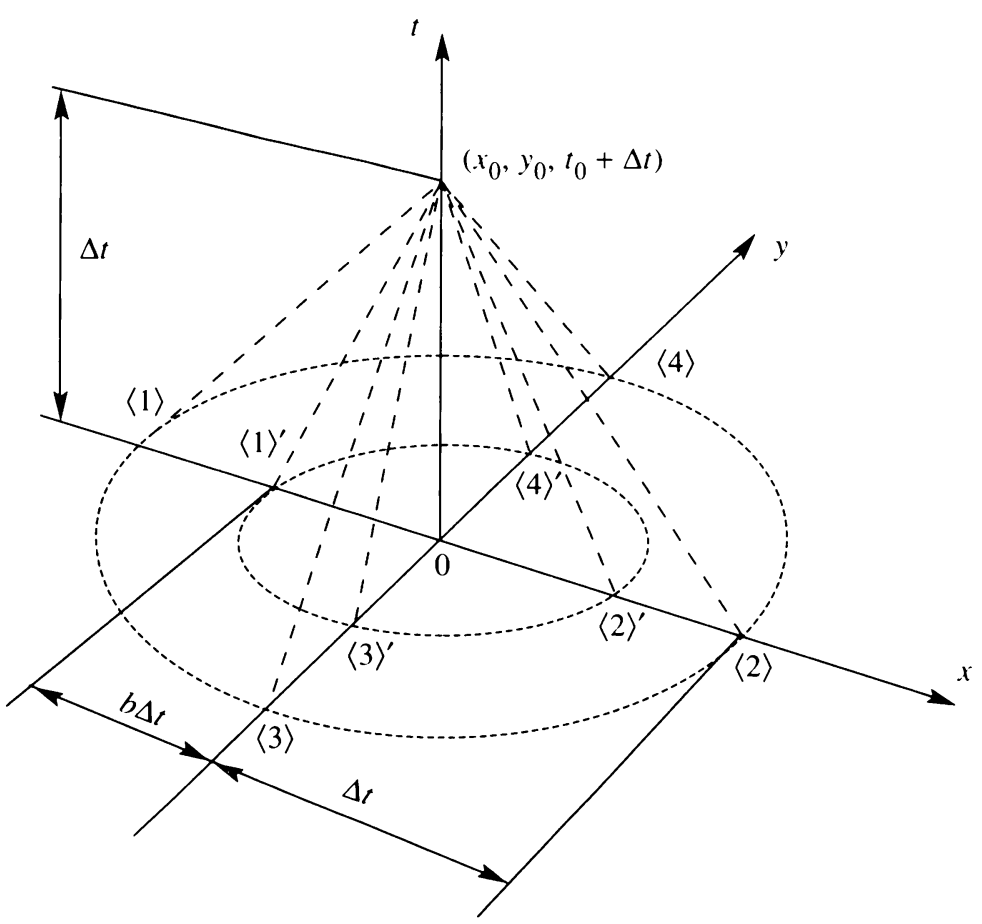

FIG. 1. A sketch of bicharacteristic lines in $(x, y, t)$ space 
Then, the second-order accurate integration of the compatibility relations (9) and (10) yields the following eight algebraic equations:

$$
\begin{gathered}
u-p-\frac{\Delta t}{2} \frac{\partial \tau}{\partial y}+\frac{a \Delta t}{2} \frac{\partial v}{\partial y}=u_{\langle 1\rangle}-p_{\langle 1\rangle}+\frac{\Delta t}{2}\left(\frac{\partial \tau}{\partial y}\right)_{\langle 1\rangle}-\frac{a \Delta t}{2}\left(\frac{\partial v}{\partial y}\right)_{\langle 1\rangle}, \\
u+p-\frac{\Delta t}{2} \frac{\partial \tau}{\partial y}-\frac{a \Delta t}{2} \frac{\partial v}{\partial y}=u_{\langle 2\rangle}+p_{\langle 2\rangle}+\frac{\Delta t}{2}\left(\frac{\partial \tau}{\partial y}\right)_{\langle 2\rangle}+\frac{a \Delta t}{2}\left(\frac{\partial v}{\partial y}\right)_{\langle 2\rangle}^{\prime}, \\
v-\frac{1}{b} \tau-\frac{\Delta t}{2} \frac{\partial q}{\partial y}+\frac{b \Delta t}{2} \frac{\partial u}{\partial y}=v_{\langle 1\rangle}^{\prime}-\frac{1}{b} \tau_{\langle 1\rangle}^{\prime}+\frac{\Delta t}{2}\left(\frac{\partial q}{\partial y}\right)_{\langle 1\rangle}^{\prime}-\frac{b \Delta t}{2}\left(\frac{\partial u}{\partial y}\right)_{\langle 1\rangle}^{\prime} \\
v+\frac{1}{b} \tau-\frac{\Delta t}{2} \frac{\partial q}{\partial y}-\frac{b \Delta t}{2} \frac{\partial u}{\partial y}=v_{\langle 2\rangle}^{\prime}+\frac{1}{b} \tau_{\langle 2\rangle}^{\prime}+\frac{\Delta t}{2}\left(\frac{\partial q}{\partial y}\right)_{\langle 2\rangle}^{\prime}+\frac{b \Delta t}{2}\left(\frac{\partial u}{\partial y}\right)_{\langle 2\rangle}^{\prime} \\
v-q-\frac{\Delta t}{2} \frac{\partial \tau}{\partial x}+\frac{a \Delta t}{2} \frac{\partial u}{\partial x}=v_{\langle 3\rangle}-q_{\langle 3\rangle}+\frac{\Delta t}{2}\left(\frac{\partial \tau}{\partial x}\right)_{\langle 3\rangle}-\frac{a \Delta t}{2}\left(\frac{\partial u}{\partial x}\right)_{\langle 3\rangle}, \\
v+q-\frac{\Delta t}{2} \frac{\partial \tau}{\partial x}-\frac{a \Delta t}{2} \frac{\partial u}{\partial x}=v_{\langle 4\rangle}+q_{\langle 4\rangle}+\frac{\Delta t}{2}\left(\frac{\partial \tau}{\partial x}\right)_{\langle 4\rangle}+\frac{a \Delta t}{2}\left(\frac{\partial u}{\partial x}\right)_{\langle 4\rangle}, \\
u-\frac{1}{b} \tau-\frac{\Delta t}{2} \frac{\partial p}{\partial x}+\frac{b \Delta t}{2} \frac{\partial v}{\partial x}=u_{\langle 3\rangle}^{\prime}-\frac{1}{b} \tau_{\langle 3\rangle}^{\prime}+\frac{\Delta t}{2}\left(\frac{\partial p}{\partial x}\right)_{\langle 3\rangle}^{\prime}-\frac{b \Delta t}{2}\left(\frac{\partial v}{\partial x}\right)_{\langle 3\rangle}^{\prime} \\
u+\frac{1}{b} \tau-\frac{\Delta t}{2} \frac{\partial p}{\partial x}-\frac{b \Delta t}{2} \frac{\partial v}{\partial x}=u_{\langle 4\rangle}^{\prime}+\frac{1}{b} \tau_{\langle 4\rangle}^{\prime}+\frac{\Delta t}{2}\left(\frac{\partial p}{\partial x}\right)_{\langle 4\rangle}^{\prime}+\frac{b \Delta t}{2}\left(\frac{\partial v}{\partial x}\right)_{\langle 4\rangle}^{\prime}
\end{gathered}
$$

where $u, p, \frac{\partial p}{\partial x}, \frac{\partial \tau}{\partial y}, \ldots$ on the left-hand sides of the equations are the unknown values at point $\left(x_{0}, y_{0}, t_{0}+\Delta t\right)$. The number of unknown values in Eqs. (12) is 13, which is larger than the number of equations. Therefore, Eq. (1) is integrated along the line: $x=x_{0}, y=y_{0}, t_{0} \leq t \leq t_{0}+\Delta t$ to obtain another five algebraic equations:

$$
\mathbf{w}-\frac{\Delta t}{2} \mathbf{A} \frac{\partial \mathbf{w}}{\partial x}-\frac{\Delta t}{2} \mathbf{B} \frac{\partial \mathbf{w}}{\partial y}=\mathbf{w}_{\langle 0\rangle}+\frac{\Delta t}{2} \mathbf{A}\left(\frac{\partial \mathbf{w}}{\partial x}\right)_{\langle 0\rangle}+\frac{\Delta t}{2} \mathbf{B}\left(\frac{\partial \mathbf{w}}{\partial y}\right)_{\langle 0\rangle},
$$

where $\langle 0\rangle$ (and below $\langle 0\rangle^{\prime}$ in Eq. (15)) represents the point $\left(x_{0}, y_{0}, t_{0}\right)$. Thus, Eqs. (12) and (13) can be solved simultaneously for the 13 unknown values. As results, only the five components of $\mathbf{w}$ are of interest in the numerical calculation. We define $\widehat{\mathbf{A}}$ and $\widehat{\mathbf{B}}$ by

$$
\widehat{\mathbf{A}}=\left(\begin{array}{lllll}
0 & 0 & 1 & 0 & 0 \\
0 & 0 & 0 & 0 & 0 \\
1 & 0 & 0 & 0 & 0 \\
a & 0 & 0 & 0 & 0 \\
0 & 0 & 0 & 0 & 0
\end{array}\right), \quad \widehat{\mathbf{B}}=\left(\begin{array}{ccccc}
0 & 0 & 0 & 0 & 0 \\
0 & 0 & 0 & 1 & 0 \\
0 & a & 0 & 0 & 0 \\
0 & 1 & 0 & 0 & 0 \\
0 & 0 & 0 & 0 & 0
\end{array}\right)
$$


Then, $\mathbf{w}$ can be expressed in the following matrix form:

$$
\begin{aligned}
\mathbf{w}= & \mathbf{w}_{\langle 0\rangle}+\frac{1}{2} \widehat{\mathbf{A}}\left(\mathbf{w}_{\langle 2\rangle}-\mathbf{w}_{\langle 1\rangle}\right)+\frac{1}{2 b}(\mathbf{A}-\widehat{\mathbf{A}})\left(\mathbf{w}_{\langle 2\rangle}^{\prime}-\mathbf{w}_{\langle 1\rangle}^{\prime}\right) \\
& +\frac{1}{2} \widehat{\mathbf{B}}\left(\mathbf{w}_{\langle 4\rangle}-\mathbf{w}_{\langle 3\rangle}\right)+\frac{1}{2 b}(\mathbf{B}-\widehat{\mathbf{B}})\left(\mathbf{w}_{\langle 4\rangle}^{\prime}-\mathbf{w}_{\langle 3\rangle}^{\prime}\right) \\
& +\frac{1}{2} \widehat{\mathbf{A}}^{2}\left(\mathbf{w}_{\langle 2\rangle}-2 \mathbf{w}_{\langle 0\rangle}+\mathbf{w}_{\langle 1\rangle}\right)+\frac{1}{2 b^{2}}\left(\mathbf{A}^{2}-\widehat{\mathbf{A}}^{2}\right)\left(\mathbf{w}_{\langle 2\rangle}^{\prime}-2 \mathbf{w}_{\langle 0\rangle}^{\prime}+\mathbf{w}_{\langle 1\rangle}^{\prime}\right) \\
& +\frac{1}{2} \widehat{\mathbf{B}}^{2}\left(\mathbf{w}_{\langle 4\rangle}-2 \mathbf{w}_{\langle 0\rangle}+\mathbf{w}_{\langle 3\rangle}\right)+\frac{1}{2 b^{2}}\left(\mathbf{B}^{2}-\widehat{\mathbf{B}}^{2}\right)\left(\mathbf{w}_{\langle 4\rangle}^{\prime}-2 \mathbf{w}_{\langle 0\rangle}^{\prime}+\mathbf{w}_{\langle 3\rangle}^{\prime}\right) \\
& +\frac{\Delta t}{4} \widehat{\mathbf{A}} \mathbf{B}\left[\left(\frac{\partial \mathbf{w}}{\partial y}\right)_{\langle 2\rangle}-\left(\frac{\partial \mathbf{w}}{\partial y}\right)_{\langle 1\rangle}\right]+\frac{\Delta t}{4 b}(\mathbf{A}-\widehat{\mathbf{A}}) \mathbf{B}\left[\left(\frac{\partial \mathbf{w}}{\partial y}\right)_{\langle 2\rangle}^{\prime}-\left(\frac{\partial \mathbf{w}}{\partial y}\right)_{\langle 1\rangle}^{\prime}\right] \\
& +\frac{\Delta t}{4} \widehat{\mathbf{B}} \mathbf{A}\left[\left(\frac{\partial \mathbf{w}}{\partial x}\right)_{\langle 4\rangle}-\left(\frac{\partial \mathbf{w}}{\partial x}\right)_{\langle 3\rangle}\right]+\frac{\Delta t}{4 b}(\mathbf{B}-\widehat{\mathbf{B}}) \mathbf{A}\left[\left(\frac{\partial \mathbf{w}}{\partial x}\right)_{\langle 4\rangle}^{\prime}-\left(\frac{\partial \mathbf{w}}{\partial x}\right)_{\langle 3\rangle}^{\prime}\right] \\
& +\frac{\Delta t}{4} \widehat{\mathbf{A}}^{2} \mathbf{B}\left[\left(\frac{\partial \mathbf{w}}{\partial y}\right)_{\langle 2\rangle}-2\left(\frac{\partial \mathbf{w}}{\partial y}\right)_{\langle 0\rangle}+\left(\frac{\partial \mathbf{w}}{\partial y}\right)_{\langle 1\rangle}\right] \\
& +\frac{\Delta t}{4 b^{2}}\left(\mathbf{A}^{2}-\widehat{\mathbf{A}}^{2}\right) \mathbf{B}\left[\left(\frac{\partial \mathbf{w}}{\partial y}\right)_{\langle 2\rangle}^{\prime}-2\left(\frac{\partial \mathbf{w}}{\partial y}\right)_{\langle 0\rangle}^{\prime}+\left(\frac{\partial \mathbf{w}}{\partial y}\right)_{\langle 1\rangle}^{\prime}\right] \\
& +\frac{\Delta t}{4} \widehat{\mathbf{B}}^{2} \mathbf{A}\left[\left(\frac{\partial \mathbf{w}}{\partial x}\right)_{\langle 4\rangle}-2\left(\frac{\partial \mathbf{w}}{\partial x}\right)_{\langle 0\rangle}+\left(\frac{\partial \mathbf{w}}{\partial x}\right)_{\langle 3\rangle}\right] \\
& +\frac{\Delta t}{4 b^{2}}\left(\mathbf{B}^{2}-\widehat{\mathbf{B}}^{2}\right) \mathbf{A}\left[\left(\frac{\partial \mathbf{w}}{\partial x}\right)_{\langle 4\rangle}^{\prime}-2\left(\frac{\partial \mathbf{w}}{\partial x}\right)_{\langle 0\rangle}^{\prime}+\left(\frac{\partial \mathbf{w}}{\partial x}\right)_{\langle 3\rangle}^{\prime}\right] .
\end{aligned}
$$

Equation (15) is the general expression for the second-order accurate numerical bicharacteristic solution of the elastodynamic equations.

3. Construction of numerical schemes. Since we consider an isotropic material, it is convenient to introduce a quadratic mesh in space with the mesh size $\Delta h \equiv \Delta x=\Delta y$. If we want to apply an explicit bicharacteristic scheme to calculate the solution in a point in space at the level $t_{0}+\Delta t$ from known data at the time level $t_{0}$, we always have to fulfill the necessary stability condition $\mathrm{CFL} \leq 1$. For $\mathrm{CFL}<1$, the eight backward characteristics will meet the plane $t=t_{0}$ in the interior part of the $2 \Delta h \times 2 \Delta h$ quadratic subdomain around the point, where we want to determine the solution at time $t_{0}+\Delta t$. But only in the $3 \times 3$ vertices, which are numbered by $0,1,2, \ldots, 8$ in Fig. 2 , the discrete values of the solutions are known. In order to calculate the initial values at the penetration points of the bicharacteristics, some kind of analytic reconstruction of the solution from its discrete values or an interpolation is needed.

If we suppose that the data at time $t=t_{0}$ are locally smooth and twice differentiable, $\mathbf{w}_{\langle\cdot\rangle}$ can be calculated by a Taylor expansion. This method, as a matter of fact, was used by Clifton in [1]. Introducing the CFL number $\lambda=\Delta t / \Delta h$, it follows 


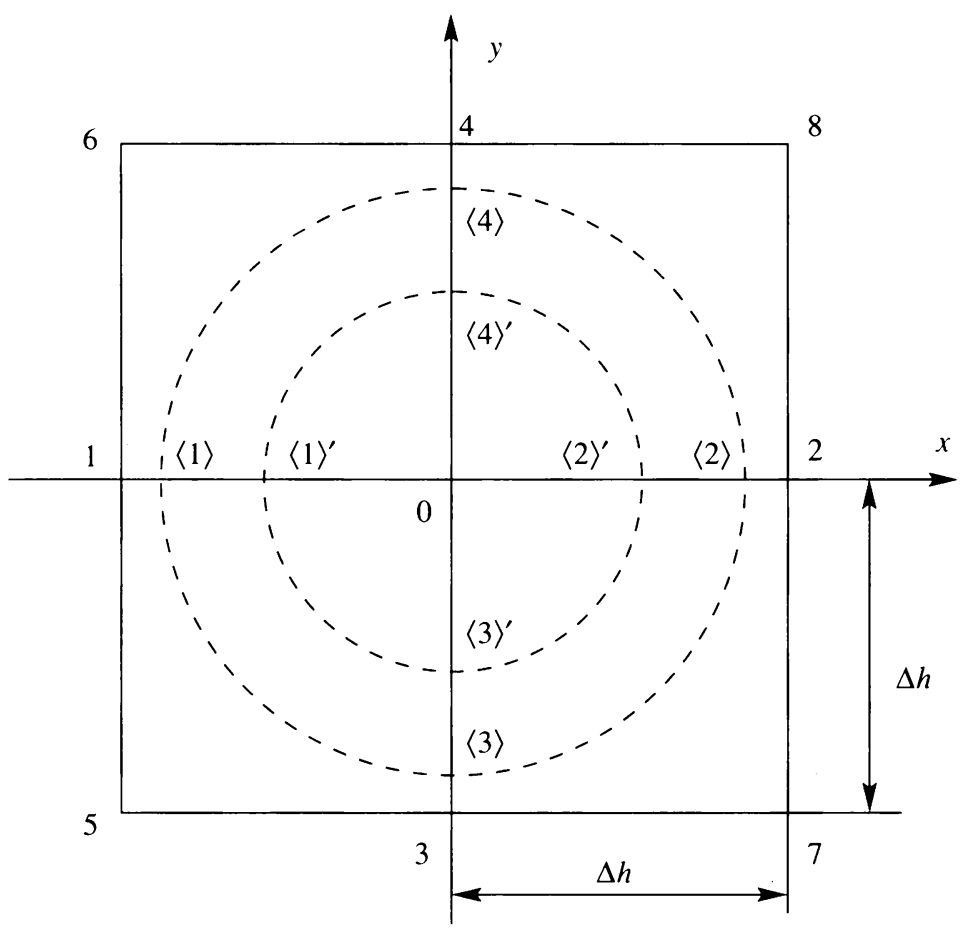

FIG. 2. A sketch of grid point positions for the numerical scheme

that

$$
\begin{aligned}
\mathbf{w}_{\langle 1\rangle} & =\mathbf{w}_{0}-\Delta t\left(\frac{\partial \mathbf{w}}{\partial x}\right)_{0}+\frac{\Delta t^{2}}{2}\left(\frac{\partial^{2} \mathbf{w}}{\partial x^{2}}\right)_{0}+\cdots \\
& \approx \mathbf{w}_{0}-\frac{\lambda}{2}\left(\mathbf{w}_{2}-\mathbf{w}_{1}\right)+\frac{\lambda^{2}}{2}\left(\mathbf{w}_{2}-2 \mathbf{w}_{0}+\mathbf{w}_{1}\right), \\
\mathbf{w}_{\langle 1\rangle}^{\prime} & \approx \mathbf{w}_{0}-\frac{b \lambda}{2}\left(\mathbf{w}_{2}-\mathbf{w}_{1}\right)+\frac{(b \lambda)^{2}}{2}\left(\mathbf{w}_{2}-2 \mathbf{w}_{0}+\mathbf{w}_{1}\right),
\end{aligned}
$$

and so forth for the other points. Inserting these expressions into Eq. (15), we get the same form of the solution in the new point $\left(x_{0}, y_{0}, t_{0}+\Delta t\right)$ as by the Lax-Wendroff Scheme [15]:

$$
\begin{aligned}
& \mathbf{w}=\mathbf{w}_{0}+\frac{\lambda}{2}\left[\mathbf{A}\left(\mathbf{w}_{2}-\mathbf{w}_{1}\right)\right.\left.+B\left(\mathbf{w}_{4}-\mathbf{w}_{3}\right)\right] \\
&+ \frac{\lambda^{2}}{2}\left[\mathbf { A } ^ { 2 } \left(\mathbf{w}_{2}-2 \mathbf{w}_{0}\right.\right. \\
&\left.+\mathbf{w}_{1}\right)+\mathbf{B}^{2}\left(\mathbf{w}_{4}-2 \mathbf{w}_{0}+\mathbf{w}_{3}\right) \\
&\left.+\frac{1}{4}(\mathbf{A B}+\mathbf{B A})\left(\mathbf{w}_{8}-\mathbf{w}_{7}-\mathbf{w}_{6}+\mathbf{w}_{5}\right)\right]
\end{aligned}
$$

The scheme (17) was used in [1] and applied to elastic-viscoplastic problems in [612]. Unfortunately, the scheme (17) is not stable up to the values of $\lambda=1$. In order to find the upper limit of $\lambda$ for the stable solution, we consider the amplification 
matrix of scheme (17), which is denoted by $\mathbf{G}_{1}$ in the following:

$$
\begin{aligned}
\mathbf{G}_{1}= & \mathbf{I}+i \lambda(\mathbf{A} \sin \xi+\mathbf{B} \sin \eta) \\
& -\lambda^{2}\left[\mathbf{A}^{2}(1-\cos \xi)+\mathbf{B}^{2}(1-\cos \eta)+\frac{1}{2}(\mathbf{A B}+\mathbf{B A}) \sin \xi \sin \eta\right],
\end{aligned}
$$

where $|\xi| \leq \pi,|\eta| \leq \pi, i=\sqrt{-1}$. The eigenvalues of $\mathbf{G}_{1}$ are denoted by $\mu\left(\mathbf{G}_{1}\right)$. The scheme (17) is stable, if $\mathbf{G}_{1}$ satisfies the von Neumann condition for all $(\xi, \eta)$ with $|\xi| \leq \pi,|\eta| \leq \pi$, i.e., the absolute maximum of the eigenvalues $\mu$ of $\mathbf{G}_{1}$ must be less than 1: $\max \left|\mu\left(\mathbf{G}_{1}\right)\right| \leq 1$. In particular, for $(\xi, \eta)=(\pi, \pi)$,

$$
\mathbf{G}_{1}(\pi, \pi)=\left(\begin{array}{llll}
1-2 \lambda^{2}\left(1+b^{2}\right) & & & \\
& 1-2 \lambda^{2}\left(1+b^{2}\right) & & \\
& 1-2 \lambda^{2} & -2 a \lambda^{2} & \\
& -2 a \lambda^{2} & 1-2 \lambda^{2} & \\
& & & 1-4 b^{2} \lambda^{2}
\end{array}\right)
$$

which requires that $\lambda \leq 1 / \sqrt{1+b^{2}}$.

The differentials in the scheme (17), say $\partial \mathbf{w} / \partial x$ and $\partial^{2} \mathbf{w} / \partial x^{2}$, are calculated only with the values on the line between point 1 and point 2 . This is correct, if there is no gradient between this line and line 5-7 as well as line 6-8. Similar arguments hold for partial derivatives with respect to $y$ and the line from point 3 to point 4. However, if there exists a gradient, some information is lost. In this case, the differentials should include the values on the other parallel lines. For example, let

$$
\begin{aligned}
\mathbf{w}_{\langle 1\rangle} \approx & \mathbf{w}_{0}-\frac{\lambda}{2(\gamma+2)}\left[\mathbf{w}_{7}-\mathbf{w}_{5}+\gamma\left(\mathbf{w}_{2}-\mathbf{w}_{1}\right)+\mathbf{w}_{8}-\mathbf{w}_{6}\right] \\
& +\frac{\lambda^{2}}{2(\gamma+2)}\left[\mathbf{w}_{7}-2 \mathbf{w}_{3}+\mathbf{w}_{5}+\gamma\left(\mathbf{w}_{2}-2 \mathbf{w}_{0}+\mathbf{w}_{1}\right)+\mathbf{w}_{8}-2 \mathbf{w}_{4}+\mathbf{w}_{6}\right],
\end{aligned}
$$

with $\gamma \geq 1$, and similarly for $\mathbf{w}_{\langle 1\rangle}^{\prime}$ and so forth. The most interesting case is $\gamma=2$. Then Eq. (15) results in the following scheme:

$$
\begin{aligned}
\mathbf{w}= & \mathbf{w}_{0}+\frac{\lambda}{8} \mathbf{A}\left[\mathbf{w}_{7}-\mathbf{w}_{5}+2\left(\mathbf{w}_{2}-\mathbf{w}_{1}\right)+\mathbf{w}_{8}-\mathbf{w}_{6}\right] \\
& +\frac{\lambda}{8} \mathbf{B}\left[\mathbf{w}_{6}-\mathbf{w}_{5}+2\left(\mathbf{w}_{4}-\mathbf{w}_{3}\right)+\mathbf{w}_{8}-\mathbf{w}_{7}\right] \\
& +\frac{\lambda^{2}}{8} \mathbf{A}^{2}\left[\mathbf{w}_{7}-2 \mathbf{w}_{3}+\mathbf{w}_{5}+2\left(\mathbf{w}_{2}-2 \mathbf{w}_{0}+\mathbf{w}_{1}\right)+\mathbf{w}_{8}-2 \mathbf{w}_{4}+\mathbf{w}_{6}\right] \\
& +\frac{\lambda^{2}}{8} \mathbf{B}^{2}\left[\mathbf{w}_{6}-2 \mathbf{w}_{1}+\mathbf{w}_{5}+2\left(\mathbf{w}_{4}-2 \mathbf{w}_{0}+\mathbf{w}_{3}\right)+\mathbf{w}_{8}-2 \mathbf{w}_{2}+\mathbf{w}_{7}\right] \\
& +\frac{\lambda^{2}}{8}(\mathbf{A B}+\mathbf{B A})\left(\mathbf{w}_{8}-\mathbf{w}_{7}-\mathbf{w}_{6}+\mathbf{w}_{5}\right) .
\end{aligned}
$$


Denote the amplification matrix of scheme $(21)$ by $\mathbf{G}_{2}$; then

$$
\begin{gathered}
\mathbf{G}_{2}=\mathbf{I}+\frac{i \lambda}{2}[\mathbf{A} \sin \xi(1+\cos \eta)+\mathbf{B} \sin \eta(1+\cos \xi)] \\
-\frac{\lambda^{2}}{2}\left[\mathbf{A}^{2}(1-\cos \xi)(1+\cos \eta)+\mathbf{B}^{2}(1-\cos \eta)(1+\cos \xi)\right. \\
+(\mathbf{A B}+\mathbf{B A}) \sin \xi \sin \eta]
\end{gathered}
$$

It is helpful to rewrite $\mathbf{G}_{2}$ in the following form:

$$
\begin{gathered}
\mathbf{G}_{2}=\mathbf{I}+2 i \lambda \cos \frac{\xi}{2} \cos \frac{\eta}{2} \mathbf{D}-2 \lambda^{2} \mathbf{D}^{2}, \\
\mathbf{D} \equiv \mathbf{A} \sin \frac{\xi}{2} \cos \frac{\eta}{2}+\mathbf{B} \sin \frac{\eta}{2} \cos \frac{\xi}{2}
\end{gathered}
$$

Then, the eigenvalues of $\mathbf{G}_{2}$ can be represented by the eigenvalues of $\mathbf{D}$,

$$
\mu\left(\mathbf{G}_{2}\right)=1+2 i \lambda \cos \frac{\xi}{2} \cos \frac{\eta}{2} \mu(\mathbf{D})-2 \lambda^{2}[\mu(\mathbf{D})]^{2} .
$$

The eigenvalues of $\mathbf{D}$ are easily calculated as

$$
0 ; \quad \pm \sqrt{\frac{1}{2}(1-\cos \xi \cos \eta)} ; \quad \pm b \sqrt{\frac{1}{2}(1-\cos \xi \cos \eta)}
$$

Then, the eigenvalues of $\mathbf{G}_{2}$ are

$$
\begin{gathered}
1 ; \quad 1-\lambda^{2}(1-\cos \xi \cos \eta) \pm i \lambda \cos \frac{\xi}{2} \cos \frac{\eta}{2} \sqrt{2(1-\cos \xi \cos \eta)} \\
1-(b \lambda)^{2}(1-\cos \xi \cos \eta) \pm i b \lambda \cos \frac{\xi}{2} \cos \frac{\eta}{2} \sqrt{2(1-\cos \xi \cos \eta)}
\end{gathered}
$$

which show $\max \left|\mu\left(\mathbf{G}_{2}\right)\right| \leq 1$ for all $(\xi, \eta)$ and $\lambda \leq 1$. Therefore, the CFL number equal to 1 can be used in scheme $(21)$.

One of the advantages of the scheme (21) is that it can be split up into two steps. If we denote $\mathbf{w}_{k l}^{n}=\mathbf{w}(k \Delta x, l \Delta y, n \Delta t)$ as the value at grid point $(k, l)$ at time level $t^{n}$, and start with Eq. (2) in place of Eq. (1), we obtain

$$
\begin{aligned}
\mathbf{w}_{k+1 / 2, l+1 / 2}^{n+1 / 2}= & \frac{1}{4}\left(\mathbf{w}_{k l}^{n}+\mathbf{w}_{k+1, l}^{n}+\mathbf{w}_{k, l+1}^{n}+\mathbf{w}_{k+1, l+1}^{n}\right) \\
& +\frac{\lambda}{4}\left(\mathbf{f}_{k+1, l}^{n}-\mathbf{f}_{k l}^{n}+\mathbf{f}_{k+1, l+1}^{n}-\mathbf{f}_{k, l+1}^{n}\right) \\
& +\frac{\lambda}{4}\left(\mathbf{g}_{k, l+1}^{n}-\mathbf{g}_{k l}^{n}+\mathbf{g}_{k+1, l+1}^{n}-\mathbf{g}_{k+1, l}^{n}\right) \\
\mathbf{w}_{k l}^{n+1}= & \mathbf{w}_{k l}^{n}+\lambda\left(\mathbf{f}_{k+1 / 2, l}^{n+1 / 2}-\mathbf{f}_{k-1 / 2, l}^{n+1 / 2}\right)+\lambda\left(\mathbf{g}_{k, l+1 / 2}^{n+1 / 2}-\mathbf{g}_{k, l-1 / 2}^{n+1 / 2}\right),
\end{aligned}
$$

where $\mathbf{f}_{k+1 / 2, l}^{n+1 / 2}=\mathbf{f}\left(\left(\mathbf{w}_{k+1 / 2, l+1 / 2}^{n+1 / 2}+\mathbf{w}_{k+1 / 2, l-1 / 2}^{n+1 / 2}\right) / 2\right)$, etc. Equations (27) were first formulated by Eilon, Gottlieb, and Zwas [16] for problems in gas dynamics. With a two-step scheme, nonlinear problems can be treated with less difficulty. In [13, 1718], Eqs. (27) have been extended for the elastic-plastic case by solving a Riemann problem for the fluxes $\mathbf{f}_{k+1 / 2, l+1 / 2}^{n+1 / 2}$ and $\mathbf{g}_{k+1 / 2, l+1 / 2}^{n+1 / 2}$. 
There is another technique for obtaining $\mathbf{w}_{\langle\cdot\rangle}$ and its related scheme. Suppose $\left(x_{0}, y_{0}\right)=(0,0)$ and $\mathbf{w}\left(x, y, t_{0}\right)$ can be approximated by a quadratic polynomial:

$$
\mathbf{w}\left(x, y, t_{0}\right)=\mathbf{c}^{(0)}+\frac{x}{\Delta h} \mathbf{c}^{(1)}+\frac{y}{\Delta h} \mathbf{c}^{(2)}+\frac{x^{2}}{\Delta h^{2}} \mathbf{c}^{(3)}+\frac{y^{2}}{\Delta h^{2}} \mathbf{c}^{(4)}+\frac{2 x y}{\Delta h^{2}} \mathbf{c}^{(5)} .
$$

When $\mathbf{w}_{\langle\cdot\rangle}$ is calculated by Eq. (28), Eq. (15) yields the following scheme:

$$
\begin{aligned}
\mathbf{w}= & \mathbf{c}^{(0)}+\lambda\left(\mathbf{A} \mathbf{c}^{(1)}+\mathbf{B c}^{(2)}\right) \\
& +\lambda^{2}\left[\mathbf{A}^{2} \mathbf{c}^{(3)}+\mathbf{B}^{2} \mathbf{c}^{(4)}+(\mathbf{A B}+\mathbf{B A}) \mathbf{c}^{(5)}\right] .
\end{aligned}
$$

The coefficients $\mathbf{c}^{(\cdot)}$ will be determined by the least squares method using the $3 \times 3$ sets of discrete values at the grid points. Substituting the coordinates $\left(x_{j}, y_{j}\right)$ and the values $\mathbf{w}_{j}$ into Eq. (28),

$$
\begin{aligned}
&\left(1, \frac{x_{j}}{\Delta h}, \frac{y_{j}}{\Delta h}, \frac{x_{j}^{2}}{\Delta h^{2}}, \frac{y_{j}^{2}}{\Delta h^{2}}, \frac{2 x_{j} y_{j}}{\Delta h^{2}}\right) \mathbf{C}=\mathbf{w}_{j} \\
&(j=0,1, \ldots, 8),
\end{aligned}
$$

where $\mathbf{C}=\left(\mathbf{c}^{(0)}, \mathbf{c}^{(1)}, \mathbf{c}^{(2)}, \mathbf{c}^{(3)}, \mathbf{c}^{(4)}, \mathbf{c}^{(5)}\right)^{\mathrm{T}}$. We rewrite Eqs. (30) into the following form:

$$
\mathbf{H C}=\mathbf{W},
$$

with $\mathbf{H}$ a $9 \times 6$ matrix and $\mathbf{W}=\left(\mathbf{w}_{0}, \mathbf{w}_{1}, \ldots, \mathbf{w}_{8}\right)^{\mathrm{T}}$. The solution of the least squares method for Eq. (31) is

$$
\mathbf{C}=\left(\mathbf{H}^{\mathrm{T}} \mathbf{H}\right)^{-1} \mathbf{H}^{\mathrm{T}} \mathbf{W} .
$$

In the above-mentioned least squares procedure, the contributions from all nine grid points seem to be equal. In a general case, the contributions can be taken to be different by using weighting functions. Suppose that the weighting functions are distributed over nine grid points in the following manner (in accordance with the grid positions in Fig. 2):

$$
\left\{\begin{array}{lll}
1 & \beta & 1 \\
\beta & \alpha & \beta \\
1 & \beta & 1
\end{array}\right\} .
$$

This means that the first equation $(j=0)$ in Eqs. (30) is multiplied by $\alpha$, and the next four equations $(j=1,2,3,4)$ are multiplied by $\beta$. The functions $\alpha$ and $\beta$ are always chosen to satisfy $\alpha \geq \beta \geq 1$, which implies that the center grid point makes the highest contribution, and grid points far away from the center make less contribution. Therefore, the solution $\mathbf{C}$ takes the form

$$
\mathbf{C}=\left(\mathbf{H}^{\mathrm{T}} \Lambda \mathbf{H}\right)^{-1} \mathbf{H}^{\mathrm{T}} \Lambda \mathbf{W},
$$

where $\Lambda=\operatorname{diag}(\alpha, \beta, \beta, \beta, \beta, 1,1,1,1)$ is a $9 \times 9$ diagonal matrix. It is fortunate that the matrix $\mathbf{H}^{\mathrm{T}} \Lambda \mathbf{H}$ is simple; then the components of $\mathrm{C}$ can be resolved 
as

$$
\begin{aligned}
& \mathbf{c}^{(0)}=\frac{1}{\Gamma}\left[\alpha(\beta+4) \mathbf{w}_{0}+2 \beta\left(\mathbf{w}_{1}+\mathbf{w}_{2}+\mathbf{w}_{3}+\mathbf{w}_{4}\right)-\beta\left(\mathbf{w}_{5}+\mathbf{w}_{6}+\mathbf{w}_{7}+\mathbf{w}_{8}\right)\right], \\
& \mathbf{c}^{(1)}=\frac{1}{2(\beta+2)}\left[\beta\left(\mathbf{w}_{2}-\mathbf{w}_{1}\right)+\mathbf{w}_{7}-\mathbf{w}_{5}+\mathbf{w}_{8}-\mathbf{w}_{6}\right], \\
& \mathbf{c}^{(2)}=\frac{1}{2(\beta+2)}\left[\beta\left(\mathbf{w}_{4}-\mathbf{w}_{3}\right)+\mathbf{w}_{6}-\mathbf{w}_{5}+\mathbf{w}_{8}-\mathbf{w}_{7}\right], \\
& \quad \mathbf{c}^{(3)}=\frac{1}{2 \Gamma}\left[\alpha(\beta+2)\left(\mathbf{w}_{2}-2 \mathbf{w}_{0}+\mathbf{w}_{1}\right)\right. \\
& \mathbf{c}^{(4)}=\frac{1}{2 \Gamma}\left[\alpha(\beta+2)\left(\mathbf{w}_{4}-2 \mathbf{w}_{0}+\mathbf{w}_{3}\right)\right. \\
& \left.\quad+(\alpha+2 \beta)\left(\mathbf{w}_{6}-2 \mathbf{w}_{1}+\mathbf{w}_{5}+\mathbf{w}_{8}-2 \mathbf{w}_{2}+\mathbf{w}_{7}\right)\right], \\
& \mathbf{c}^{(5)}=\frac{1}{8}\left(\mathbf{w}_{8}-\mathbf{w}_{7}-\mathbf{w}_{6}+\mathbf{w}_{5}\right),
\end{aligned}
$$

where $\Gamma=\alpha \beta+4 \alpha+4 \beta$.

Weighting functions have been used to calculate the results of [2-5]. In order to ensure the stability condition, the authors of those papers have tested some parameters. They have obtained one successful result in which the parameters are represented as a function of the CFL number $\lambda$ and the ratio of two wave speeds $b=c_{2} / c_{1}$ :

$$
\alpha=\frac{(\sqrt{2}+1) \lambda-\sqrt{\left(1+b^{2}\right) / 2}}{\lambda-\sqrt{\left(1+b^{2}\right) / 2}}, \quad \beta=\frac{(\sqrt{2}+1) \lambda-\sqrt{\left(1+b^{2}\right) / 2}}{2 \lambda-\sqrt{\left(1+b^{2}\right) / 2}} .
$$

Obviously the derivation of Eqs. (35) admits many more possible choices for $\alpha$ and $\beta$.

The most interesting case is $\alpha=4, \beta=2$. In this case Eqs. (29) and (35) lead to the following numerical scheme:

$$
\begin{aligned}
\mathbf{w}= & \frac{1}{16}\left[12 \mathbf{w}_{0}+2\left(\mathbf{w}_{1}+\mathbf{w}_{2}+\mathbf{w}_{3}+\mathbf{w}_{4}\right)-\left(\mathbf{w}_{5}+\mathbf{w}_{6}+\mathbf{w}_{7}+\mathbf{w}_{8}\right)\right] \\
& +\frac{\lambda}{8} \mathbf{A}\left[\mathbf{w}_{7}-\mathbf{w}_{5}+2\left(\mathbf{w}_{2}-\mathbf{w}_{1}\right)+\mathbf{w}_{8}-\mathbf{w}_{6}\right] \\
& +\frac{\lambda}{8} \mathbf{B}\left[\mathbf{w}_{6}-\mathbf{w}_{5}+2\left(\mathbf{w}_{4}-\mathbf{w}_{3}\right)+\mathbf{w}_{8}-\mathbf{w}_{7}\right] \\
& +\frac{\lambda^{2}}{8} \mathbf{A}^{2}\left[\mathbf{w}_{7}-2 \mathbf{w}_{3}+\mathbf{w}_{5}+2\left(\mathbf{w}_{2}-2 \mathbf{w}_{0}+\mathbf{w}_{1}\right)+\mathbf{w}_{8}-2 \mathbf{w}_{4}+\mathbf{w}_{6}\right] \\
& +\frac{\lambda^{2}}{8} \mathbf{B}^{2}\left[\mathbf{w}_{6}-2 \mathbf{w}_{1}+\mathbf{w}_{5}+2\left(\mathbf{w}_{4}-2 \mathbf{w}_{0}+\mathbf{w}_{3}\right)+\mathbf{w}_{8}-2 \mathbf{w}_{2}+\mathbf{w}_{7}\right] \\
& +\frac{\lambda^{2}}{8}(\mathbf{A B}+\mathbf{B A})\left(\mathbf{w}_{8}-\mathbf{w}_{7}-\mathbf{w}_{6}+\mathbf{w}_{5}\right) .
\end{aligned}
$$

Scheme (37) is different from scheme (21) only in the first term of the right-hand side. Therefore, it can also be split up into two steps. The amplification matrix of 
scheme $(37)$ is

$$
\mathbf{G}_{3}=\frac{1}{4}(3+\cos \xi+\cos \eta-\cos \xi \cos \eta) \mathbf{I}+2 i \lambda \cos \frac{\xi}{2} \cos \frac{\eta}{2} \mathbf{D}-2 \lambda^{2} \mathbf{D}^{2},
$$

where $\mathbf{D}$ is the same as in Eqs. (23). It is then not difficult to calculate the eigenvalues of $\mathbf{G}_{3}$ and to know that the von Neumann condition can be satisfied for $\lambda \leq 1$.

However, it should be noticed that not all values of $\alpha$ and $\beta$ for Eqs. (35) are acceptable. For example, take $\alpha=\beta^{2}$ and let $\beta \rightarrow \infty$; then scheme (29) with (35) will become the same as scheme (17), in which $\lambda$ cannot be set to 1 . For the antiplane shear problem [13], $\mathbf{A}$ and $\mathbf{B}$ are $3 \times 3$ matrices, in which eigenvalues can be solved explicitly. In this case, the stability region is $\alpha<\infty, \beta \leq 2.6$.

4. A numerical test for the singular point problem. As pointed out in [13], a reasonable finite difference scheme to deal with stress waves in elastic-plastic solids should possess three properties: (i) its CFL number can be chosen up to the limiting value 1 in order to minimize the numerical dissipation and dispersion; (ii) it produces few numerical defects if a singular point is present, so that wave interactions with a crack tip can be calculated appropriately; (iii) it contains two steps so that plastic yielding may be included in the physical problem, and the Riemann solver can be applied. It was already shown in the last section that the numerical schemes (21) and (37) possess the properties (i) and (iii). But for (ii), the two schemes exhibit a different solution behaviour.

Let us consider the problem of a mode I central crack in an infinite body, which was discussed in [19-20] with analytical methods. A numerical modelling of this problem can be found in [21]. The problem is shown in Fig. 3(a), where two plane waves of equal amplitude arrive simultaneously at the crack from both sides $y>0$ and $y<0$. The signs of $v$ are opposite and the wave fronts are parallel to the crack surface. Because of the symmetry, the $x$-and $y$-axes can be introduced as boundaries such that only the solution in the first quadrant has to be calculated; see Fig. 3(b). The material constant $b$ is set to $b=1 / \sqrt{3}$. In order to ensure that the

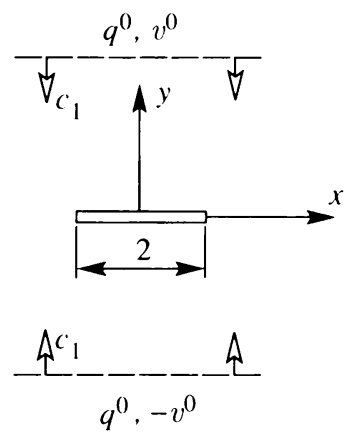

(a)

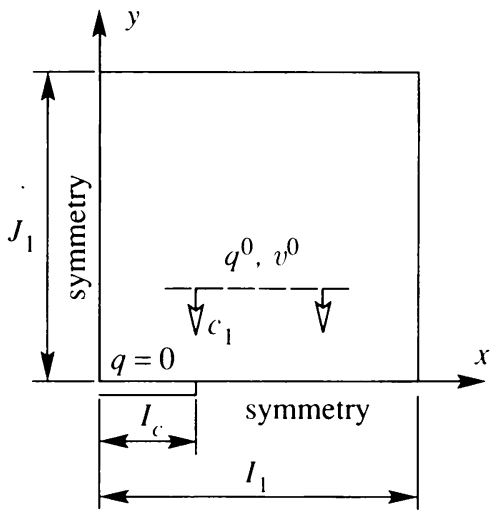

(b)

FIG. 3. Sketches of the calculated crack problem. (a) Physical problem; (b) Zoning for calculation 
stress field at the crack tip region can be calculated in a large time interval without being disturbed by the reflected wave from outer boundaries, the number of grid points is chosen as $I_{c}=20, I_{1}=200, J_{1}=180$. The $x$-axis is located at the middle of two rows of grids, and so too is the $y$-axis. Since the schemes (21) and (37) can be divided into two steps, it is easy to introduce the boundary conditions in the first step by using the flux. A method for treating boundary conditions is given in [17] and [21], in which the cell's center corresponds to a grid point in this paper. At time $t=0$ the waves just arrive at $y=0$, and the initial conditions for all grid points are

$$
q^{0}=v^{0}=0.5, \quad u^{0}=\tau^{0}=0, \quad p^{0}=\frac{\nu}{1-\nu} q^{0},
$$

where $\nu=a /(1+a)$ is Poisson's ratio. The resulting distributions of stress $q$ and velocity $v$ at time step $N=320$ (time $=N / I_{c}=16$ ) are plotted in Figs. 4 and 5 , where method 1 and 2 represent schemes (21) and (37), respectively. The results seem quite interesting. Apart from some oscillations, method 1 delivers a good stress distribution, but exhibits some defects for the velocity component in the region near the crack tip. In contrast to that, method 2 creates a cutting trace in the stress distribution, but leads to a smooth velocity distribution.
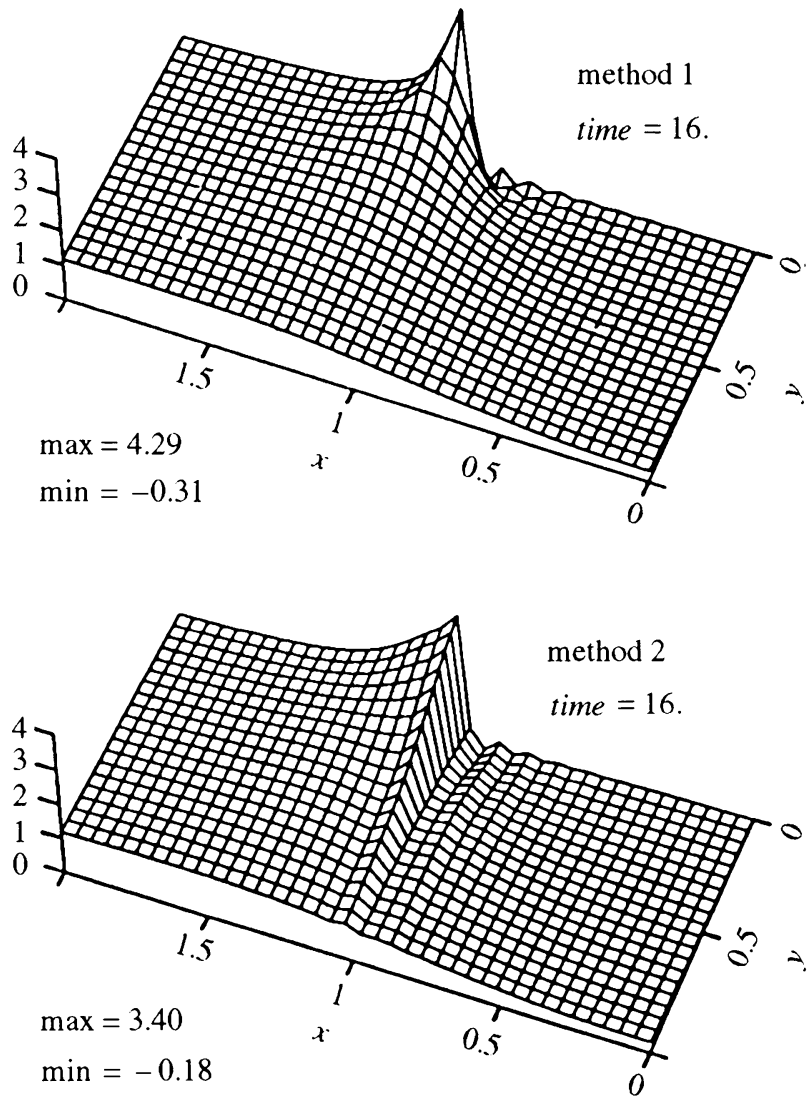

FIG. 4. Comparison of stress $q$ distribution of two numerical schemes for a crack problem 

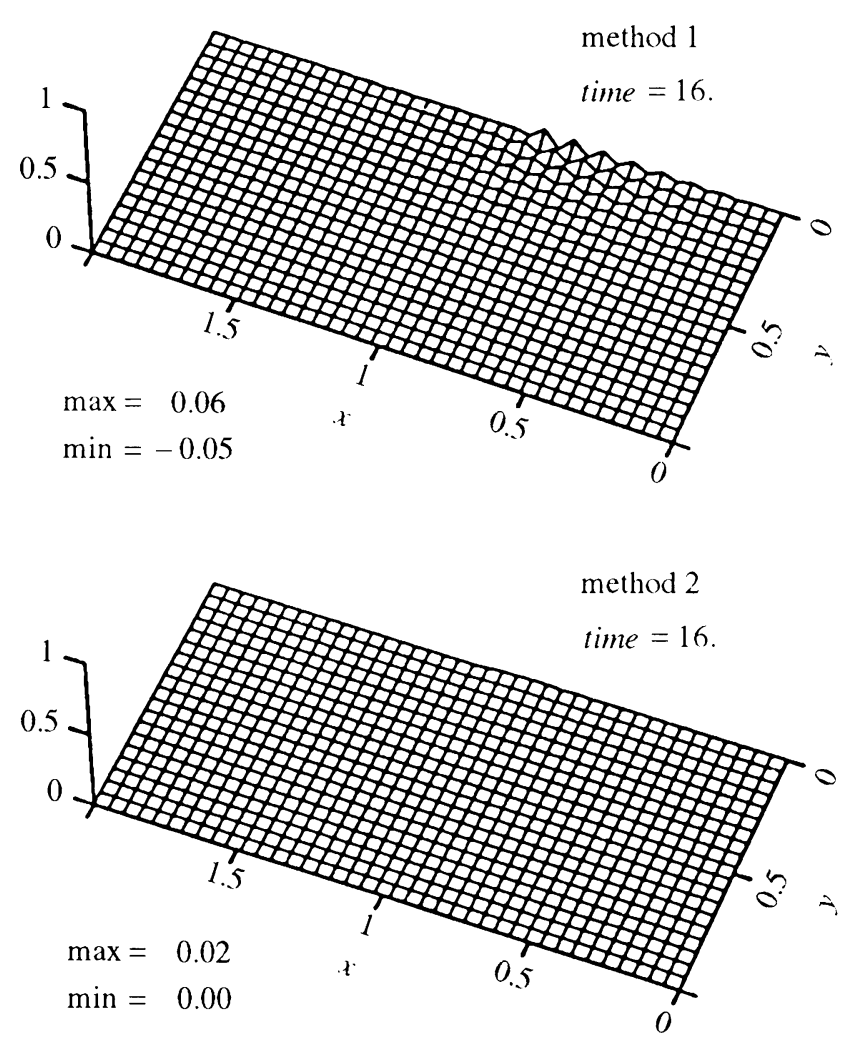

FIG. 5. Comparison of velocity $v$ distribution of two numerical schemes for a crack problem

The solution around the crack tip always shows numerical errors no matter which approximation method is used. This is due to the singularity and the high gradient of the stresses in the crack-tip region, which cannot be modeled adequately by a coarse rectangular mesh. On the other hand, the example presented above shows that the spreading of numerical errors differs. Method 1 distributes the error over a region, while method 2 concentrates it in a cutting trace. Schemes (21) and (37) are different only in the first term. In the first term of scheme (37) the contributions from points 1, 2, 3, and 4 (see Fig. 2) are strengthened, while those from points 5, 6,7 , and 8 are weakened. This term introduces a smoothing to the computation. However, in a singular point region, it will lead to a cutting trace. A cutting trace also became apparent in the examples of [5] and [12] (Fig. 4). Carrying out some numerical experiments with the combination of scheme (29) and (35) we always found a cutting trace around a singular point no matter how $\alpha$ and $\beta$ were chosen. So the comparison of the two methods gives us a good explanation for the appearance of the cutting trace.

Nevertheless, it should be pointed out that an appropriate combination of schemes (21) and (37) will create a useful method to compute dynamic crack problems. For example, scheme (21) can be used for the near crack-tip region, and scheme (37) 
for the far field; another method might be to use scheme (21) to calculate the stress components, and scheme (37) for the velocity components. For a particular problem, of course, some numerical tests are necessary to find out the optimal combination.

5. Conclusions. To conclude this paper, we make two remarks.

(i) The CFL number of an explicit finite difference scheme for hyperbolic PDEs is very important in practice, not only for the numerical stability, but also for the control of numerical dissipation and dispersion. This was also shown explicitly for one-dimensional problems in [22], where for each wave mode a proper grid was introduced such that the CFL number became equal to 1 for both longitudinal and transverse waves. Here we have presented some techniques to form two-dimensional bicharacteristic schemes in which the CFL number of longitudinal mode can be set to its limiting value 1 . Among them, schemes (21) and (37) are the most important ones, because they can be divided into two steps. Following [22] it will be possible to construct bicharacteristic schemes which admit $\mathrm{CFL}=1$ for the transverse mode for linear problems, too, by introducing two meshes.

(ii) A cutting trace is a nonphysical discontinuity appearing in the region near the crack-tip or other singular points. It can appear not only in the difference method based on the operator splitting technique, but also in some bicharacteristic schemes. The generation of the cutting trace can be explained by comparing schemes (21) and (37).

Acknowledgments. The research work presented in this paper was supported by the Deutsche Forschungsgemeinschaft under Grant No. Ba 661/12-1, which is gratefully acknowledged. We would like to thank our colleague Mr. R. Niethammer for the helpful discussion on the least squares technique based on the weighting functions.

\section{REFERENCES}

[1] R. J. Clifton, A difference method for plane problems in dynamic elasticity, Quart. Appl. Math. 25, $9 /-116(1967)$

[2] J. Ballmann, H. J. Raatschen, and M. Staat, High stress intensities in focussing zones of waves, Local Effects in the Analysis of Structures (P. Ladeveze, ed.), Elsevier, Amsterdam, 1985, pp. 235-252

[3] J. Ballmann and M. Staat, Computation of impacts on elastic solids by methods of bicharacteristics, Computational Mechanics '88, Theory and Applications (S. N. Atluri and G. Yagawa, eds.), Vol. 2, Springer-Verlag, New York, 1988, Chapter 60, pp. i1-i4

[4] J. Ballmann and K.-S. Kim, Numerische Simulation mechanischer Wellen in geschichteten elastischen Körpern, Z. Angew. Math. Mech. 70, T204-T206 (1990)

[5] K.-S. Kim, Spannungswellen an Grenzflächen in linearelastischen Scheiben, thesis for doctorate RWTH Aachen, VDI Verlag, Reihe 18, Nr. 91 (1991)

[6] J. Bejda, Propagation of two-dimensional stress waves in an elastic/viscoplastic material, Proceedings of the 12th International Congress of Applied Mechanics, Stanford University, 121-134 (1968)

[7] H. Fukuoka and H. Toda, High velocity impact of mild steel cylinder, Proceedings of IUTAM Symposium, Springer-Verlag, 397-402 (1978)

[8] K. Fukatsu, K. Kawashima, and M. Oda, Dynamic elastic response of a short circular cylinder due to longitudinal impact, Trans. Japan. Soc. Mech. Engrg. (in Japanese) 50A, 869 (1984)

[9] K. Liu, S. Tanimura, H. Igaki, and K. Kaizu, The dynamic behavior of an elastic circular tube due to longitudinal impact, JSME International Journal series I 32, 535-539 (1989)

[10] K. Liu and T. Yokoyama, Dynamic behavior of elastic/viscoplastic bars of square cross section subjected to longitudinal impact, Trans. Japan. Soc. Mech. Engrg. (in Japanese) 58A, 109-116 (1992) 
[11] G. Ravichandran and R. J. Clifton, Dynamic fracture under plane wave loading, Internat. J. Fracture 40, 157-201 (1989)

[12] G. Ravichandran, An analysis of dynamic crack initiation and propagating in elastic-viscoplastic solids, ICF-7 Advances in Fracture Research, Vol. 1 (K. Salama et al., eds.), Pergamon, 1989, pp. 819-826

[13] X. Lin and J. Ballmann, Numerical method for elastic-plastic waves in cracked solids, part 1: antiplane shear problem, Archive of Applied Mechanics (Ingenieur-Archiv) 63, 261-282 (1993)

[14] G. Strang, Accurate partial difference methods in nonlinear problems, Numer. Math. 13, 37-46 (1964)

[15] P. D. Lax and B. Wendroff, Difference schemes for hyperbolic equations with high order of accuracy, Comm. Pure Appl. Math. 17, 381-398 (1964)

[16] B. Eilon, D. Gottlieb, and G. Zwas, Numerical stabilizers and computing time for second-order accurate schemes, J. Comput. Phys. 9, 387-397 (1972)

[17] X. Lin and J. Ballmann, Numerical method for elastic-plastic waves in cracked solids, part 2: plane strain problem, Archive of Applied Mechanics (Ingenieur-Archiv) 63, 283-295 (1993)

[18] X. Lin and J. Ballmann, A finite difference method for elastic-plastic waves in solids, Numerical Methods in Engineering '92 (Ch. Hirsch et al., eds.), Elsevier, Amsterdam, 1992, pp. 681-686

[19] S. A. Thau and T. H. Lu, Transient stress intensity factors for a finite crack in an elastic solid caused by a dilatational wave, Internat. J. Solids and Structures 7, 731-750 (1971)

[20] K. S. Kim, Dynamic propagation of a finite crack, Internat. J. Solids and Structures 15, 685-699 (1979)

[21] X. Lin and J. Ballmann, Re-consideration of Chen's problem by finite difference method, Engineering Fracture Mechanics 44, 735-739 (1993)

[22] H. D. Lauermann, Ein Charakteristiken-Algorithmus zur Berechnung der instationären Transversalschwingungen von Hubschrauberrotorblättern oder anderer quasi-eindimensionaler elastischer Strukturen, thesis for doctorate RWTH Aachen (1990). See also: H. D. Lauermann, Instationäre Verformungen elastischer Rotorblätter, Z. Angew. Math. Mech. 70, T213-T215 (1990) 